\title{
The Effect of State-Dependent Control for an SIRS Epidemic Model with Varying Total Population
}

\author{
Fuwei Zhang, Linfei Nie* \\ College of Mathematics and Systems Science, Xinjiang University, Urumqi, China \\ Email: ${ }^{*}$ lfnie@163.com
}

How to cite this paper: Zhang, F.W. and Nie, L.F. (2016) The Effect of State-Dependent Control for an SIRS Epidemic Model with Varying Total Population. Journal of Applied Mathematics and Physics, 4, 18891898.

http://dx.doi.org/10.4236/jamp.2016.410191

Received: September 24, 2016

Accepted: October 16, 2016

Published: October 20, 2016

Copyright $\odot 2016$ by authors and Scientific Research Publishing Inc. This work is licensed under the Creative Commons Attribution International License (CC BY 4.0).

http://creativecommons.org/licenses/by/4.0/

\begin{abstract}
Based on the mechanism of prevention and control of infectious disease, we propose, in this paper, an SIRS epidemic model with varying total population size and statedependent control, where the fraction of susceptible individuals in population is as the detection threshold value. By the Poincaré map, theory of differential inequalities and differential equation geometry, the existence and orbital stability of the diseasefree periodic solution are discussed. Theoretical results show that by state-dependent pulse vaccination we can make the proportion of infected individuals tend to zero, and control the transmission of disease in population.
\end{abstract}

\section{Keywords}

SIRS Epidemic Model, Varying Total Population, State-Dependent Pulse Control, Periodic Solution, Orbital Stability

\section{Introduction}

It is generally known that the spread of infectious diseases has been a threat to healthy of human beings and other species. In order to prevent and control the transmission of disease (such as hepatitis C, malaria, influenza), pulse vaccination as an effective strategy has been widely studied by many scholars in the study of mathematical epidemiology. In the classical research literature it is usually assumed that the pulse vaccination occurs at fixed moment intervals and total population size remains constant [1] [2], and so on. Although fixed time pulse vaccination strategy is better than the traditional vaccination strategies (continuous vaccination), it has a few disadvantages. For these reasons, a new vaccination strategies, state-dependent pulse vaccination is proposed when the number of the susceptible individuals or infected individuals reaches a critical value. Clearly, the latter control strategies are more rational for disease control because of its efficiency, economy, and feasibility. In recent 
years, mathematical models with state-dependent pulse control strategies have been extensively applied to research fields of applied science, such as pest management model [3], tumor model [4], predator-prey model [5], and others. Particularly, Nie et al. [6] investigated an SIR epidemic model with state-dependent pulse vaccination. In it, authors obtained the existence and stability of positive order- 1 and order- 2 periodic solution. Tang et al. [7] proposed an SIR epidemic model with state-dependent pulse control strategies. Authors demonstrated that the combination of pulse vaccination and treatment is optimal in terms of cost under certain conditions, and studied the existence and stability of periodic solution.

On the other hand, the population sizes of all epidemic models with state-dependent pulse control are constant. These types of models have been studied extensively since they are easier to analyze than variable population size models. Obviously, the assumption that the total population size which remains constant is reasonable if negligible mortality rate and the disease spread quickly through the population. However, it fails to hold for diseases that are endemic in communities with changing populations, and for diseases which raise the mortality rate substantially. In such situation, we can hardly expect a population remaining constant, and hence more complicated epidemic models with varying population size should be considered. In fact, studies of this type of models have been become a major topic in mathematical epidemiology. For example, an general epidemiological model with vaccination and varying total population was discussed by Yang et al. [8], in which the global dynamics of this model and it's corresponding proportionate model are investigated. The conditions between the two models in terms of disease eradication and persistence are obtained. Hui et al. [9] introduced an SEIS epidemic model with total population which is not stationary. Results are obtained in terms of three threshold which respectively determines whether or not the disease dies out and dynamics of epidemic model when births of population are throughout a year. At same time, they also discussed the existence of disease-free periodic solution when births of population are birth pulse. More related literature, we also can be found in [10] [11], and the references therein.

As far as we know, epidemic model with varying total population and state-dependent feedback control strategies had never been done in the literatures. Hence, in this paper, the dynamical behavior of an SIRS epidemic model with varying total population and state-dependent pulse control strategy is studied. The main aim is to explore how the state-dependent pulse control strategy affects the transmission of diseases. The remaining part of this paper is organized as follows. In the next section, an SIRS control model is constructed and some preliminaries are introduced, which are useful for the latter discussion. In section 3, we will focus our attention on the existence and orbital stability of disease-free periodic. Finally, some concluding remarks are presented in the last section.

\section{Models and Preliminaries}

In the study of the dynamic properties of infectious diseases, it was found that when the 
popularity of disease for a long time total population size change this factor should be considered. In this case, Busenberg et al. [12] proposed the following SIRS epidemic model with varying total population size.

$$
\left\{\begin{array}{l}
\frac{\mathrm{d} S(t)}{\mathrm{d} t}=b N(t)-d S(t)-\frac{\lambda S(t) I(t)}{N(t)}+e R(t), \\
\frac{\mathrm{d} I(t)}{\mathrm{d} t}=\frac{\lambda S(t) I(t)}{N(t)}-(d+c+\varepsilon) I(t), \\
\frac{\mathrm{d} R(t)}{\mathrm{d} t}=c I(t)-(d+e+\delta) R(t) .
\end{array}\right.
$$

Here $S(t), I(t)$, and $R(t)$ denote the numbers of susceptible, infected, and recovered individuals respectively, and $N(t)=S(t)+I(t)+R(t)$ denote the total population size at time $t$. The parameters in the model have the following features: $b$ is the per capita birth rate with the assumption that all newborns are susceptible; $d$ is the per capita disease free death rate of the population; the constants $\varepsilon$ and $\delta$ denote the excess per capita death rate of infected individuals and recovered individuals, respectively; $c$ is the per capital recovery rate of the infected individuals and $e$ is the per capita loss of immunity rate for recovered individuals. It is assumed that all susceptible group becomes infected at a rate $\lambda I(t) / N(t)$, where $\lambda>0$ is the effective per capita contract rate of infective individuals. All parameter values are assumed to be nonnegative and $b, c>0$.

Since the susceptible individuals are immunity toward certain infectious diseases in the crowd, once infected individuals get into the susceptible groups, this will lead to the outbreak of the diseases. For this reason, we propose a pulse vaccination function as follows $S\left(t^{+}\right)=(1-p) S(t)$ where $p(0<p<1)$ is the proportion by which the susceptible individuals numbers is reduced by pulse vaccination.

Taking into account pulse vaccination as state-dependent feedback control strategies, model (1) can be extend to the following state-dependent pulse differential equation.

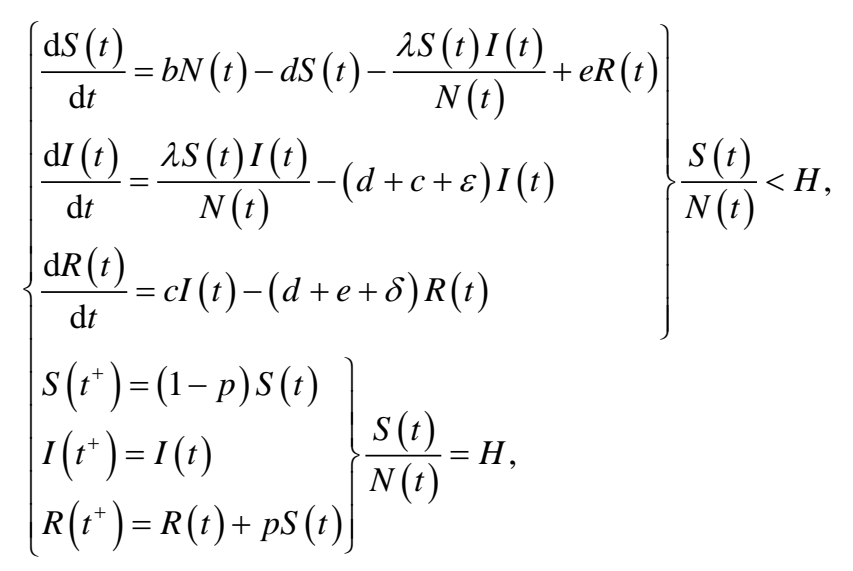

where the critical threshold $H>0$ is a constant. The meaning of model (2) as following: once the fraction of the susceptible individuals in the population reaches the critical value $H$ at time $t_{i}(H)$, vaccination control strategies are carried out which lead to the 
number of susceptible and recovered individuals abruptly turn to $(1-p) S(t)$, and $R(t)+p S(t)$ respectively.

The equation for the total population size $N(t)$ can be determined from model (2)

$$
\frac{\mathrm{d} N(t)}{\mathrm{d} t}=(b-d) N(t)-\varepsilon I(t)-\delta R(t) \neq 0 .
$$

It means that total population size $N(t)$ is not constant. In such situations, to discuss the dynamics behavior of model (2) we need to consider the fraction of individuals in the three epidemiological classes, namely

$$
x(t)=\frac{S(t)}{N(t)}, \quad y(t)=\frac{I(t)}{N(t)}, \quad z(t)=\frac{R(t)}{N(t)} .
$$

It following from (3) that we can transforms model (2) into the following model for these new variables

$$
\left\{\begin{array}{l}
\frac{\mathrm{d} x(t)}{\mathrm{d} t}=b-b x(t)+e z(t)-(\lambda-\varepsilon) x(t) y(t)+\delta x(t) z(t) \\
\frac{\mathrm{d} y(t)}{\mathrm{d} t}=\lambda x(t) y(t)+\varepsilon y^{2}(t)+\delta y(t) z(t)-(b+c+\varepsilon) y(t) \\
\frac{\mathrm{d} z(t)}{\mathrm{d} t}=c y(t)+\varepsilon y(t) z(t)+\delta z^{2}(t)-(b+e+\delta) z(t) \\
x\left(t^{+}\right)=(1-p) x(t) \\
y\left(t^{+}\right)=y(t) \\
z\left(t^{+}\right)=z(t)+p x(t)
\end{array}\right\} x(t)=H .
$$

Define three threshold parameter as follows

$$
\mathcal{R}_{0}=\lambda /(b+c+\varepsilon), \quad \mathcal{R}_{1}=\left\{\begin{array}{ll}
\frac{b}{d} & \mathcal{R}_{0} \leq 1 \\
\frac{b}{d+\varepsilon y_{e}+\delta z_{e}} & \mathcal{R}_{0}>1
\end{array}, \quad \mathcal{R}_{2}=\left\{\begin{array}{ll}
\frac{\lambda}{c+d+\varepsilon} & \mathcal{R}_{0} \leq 1 \\
\frac{\lambda x_{e}}{c+d+\varepsilon} & \mathcal{R}_{0}>1
\end{array} .\right.\right.
$$

On the dynamics of model (4) without pulse effect has been studied in [12]. Relevant conclusions can be summarized as the following Theorem 1 .

Theorem 1. For model (4) without pulse control, the following result hold true.

1) The disease-free equilibrium $E_{0}(1,0,0)$ always exists and is globally asymptotically stable in the feasibility region $\mathbb{D}=\{(x, y, z): x \geq 0, y \geq 0, z \geq 0, x+y+z \leq 1\}$ whenever $\mathcal{R}_{0} \leq 1$, and unable when $\mathcal{R}_{0}>1$.

2) When $\mathcal{R}_{0}>1$, there exist a unique endemic equilibrium $E^{*}\left(x_{e}, y_{e}, z_{e}\right)$, which is globally asymptotically stable in the feasibility region $\mathbb{D}_{0}=\mathbb{D} /\{1,0,0\}$ where

$$
x_{e}=1-y_{e}-z_{e}, \quad y_{e}=\frac{(\delta-\lambda) z_{e}+\lambda-b-c-\varepsilon}{\lambda-\varepsilon},
$$

and $z_{e}$ can be found by solving equation

$\varphi\left(z_{e}\right):=\lambda(\delta-\varepsilon) z_{e}^{2}+[-\lambda(b+c+e+\delta-\varepsilon)+\varepsilon(e-c+\delta-\varepsilon)+c \delta] z_{e}+c(\lambda-b-c-\varepsilon)=0$. 
3) The total population $N(t)$ has the asymptotic behavior $\lim _{t \rightarrow+\infty} N(t)=0$ if $\mathcal{R}_{1}<1$, and $\lim _{t \rightarrow+\infty} N(t)=+\infty$ if $\mathcal{R}_{1}>1$.

4) When $\mathcal{R}_{1}>1$, the total infected population has the asymptotic behavior $\lim _{t \rightarrow+\infty} I(t)=0$ if $\mathcal{R}_{2}<1$, and $\lim _{t \rightarrow+\infty} I(t)=+\infty$ if $\mathcal{R}_{2}>1$.

Based on the above discussions, we just need to discuss cases (a) and (b) in Table 1.

Considering the similarities of cases (a) and (b), throughout of this paper, we discuss only the case (a). That is, in a increasing population, the number of infected individuals is converges to infinity, while the fraction of infected individuals in population is tending to a nonzero constant $y_{e}$.

Due to $x(t)+y(t)+z(t)=1$, for model (4) we can eliminate $z(t)$ by $z(t)=1-x(t)-y(t)$ and consider the two-dimensional model.

$$
\left\{\begin{array}{l}
\frac{\mathrm{d} x(t)}{\mathrm{d} t}=b-b x(t)-(\lambda-\varepsilon) x(t) y(t)+(\delta x(t)+e)(1-x(t)-y(t)) \\
\frac{\mathrm{d} y(t)}{\mathrm{d} t}=\lambda x(t) y(t)+\varepsilon y^{2}(t)+\delta y(t)(1-x(t)-y(t))-(b+c+\varepsilon) y(t) \\
x\left(t^{+}\right)=(1-p) x(t) \\
y\left(t^{+}\right)=y(t)
\end{array}\right\} x(t)=H .
$$

By the biological background, we only focus on model (5) in the biological meaning region $\mathbb{D}=\{(x(t), y(t)): x(t) \geq 0, y(t) \geq 0, x(t)+y(t) \leq 1\}$. Besides, the globally existence and uniqueness properties of solution of model (5) are guaranteed by the smoothness of $f$, which is the mapping defined by right-side of model (5), for details see [13].

Let $\mathbb{S} \subset \mathbb{R}^{2}$ be an arbitrary nonempty set and $P_{0} \in \mathbb{R}^{2}$ be an arbitrary point. The distance between $P_{0}$ and $\mathbb{S}$ is defined by $\rho\left(P_{0}, \mathbb{S}\right)=\inf _{P \in \mathbb{S}}\left|P-P_{0}\right|$. Set

$X(t)=(x(t), y(t))$ be a solution of model (5) starting from initial point $X_{0} \in \mathbb{R}^{2}$ at $t=t_{0}$. We define the positive orbit as follows

$$
O^{+}\left(X_{0}, t_{0}\right)=\left\{X(t)=(x(t), y(t)): t \geq t_{0}, X\left(t_{0}\right)=X_{0}\right\} .
$$

Firstly, on the positivity of solutions of model (5), we have the following Lemma 1.

Lemma 1. Supposing that $(x(t), y(t))$ is a solution of model (5) with the initial condition $\left(x\left(t_{0}\right), y\left(t_{0}\right)\right) \in \mathbb{D}$, then $(x(t), y(t)) \in \mathbb{D}$ for all $t \geq t_{0}$.

Proof. For any initial value $\left(x\left(t_{0}\right), y\left(t_{0}\right)\right) \in \mathbb{D}$, we will discuss all possible cases by the relation of the solution $(x(t), y(t))$ to the line $L_{1}: y(t)=H$ as follows.

1) The solution $(x(t), y(t)) \in \mathbb{D}$ intersects with line $L_{1}$ finitely many times.

For this case, due to the endemic equilibrium $\left(x_{e}, y_{e}\right)$ is globally asymptotically

Table 1. Threshold criteria and asymptotic behavior.

\begin{tabular}{ccccccc}
\hline case & $\mathcal{R}_{0}$ & $\mathcal{R}_{1}$ & $\mathcal{R}_{2}$ & $N \rightarrow$ & $(x, y, z) \rightarrow$ & $(S, I, R) \rightarrow$ \\
\hline (a) & $>1$ & $>1$ & $>1$ & $+\infty$ & $\left(x_{e}, y_{e}, z_{e}\right)$ & $(+\infty,+\infty,+\infty)$ \\
(b) & $>1$ & $<1$ & $<1$ & 0 & $\left(x_{e}, y_{e}, z_{e}\right)$ & $(0,0,0)$ \\
\hline
\end{tabular}


stable, then $x(t)>0, y(t)>0$ for all $t \geq t_{0}$.

2) The solution $(x(t), y(t)) \in \mathbb{D}$ intersects with line $L_{1}$ infinitely many times.

For second situation, assume that solution $(x(t), y(t)) \in \mathbb{D}$ intersects with line $L_{1}$ at times $t_{i}(i=1,2, \cdots)$ and $\lim _{i \rightarrow \infty} t_{i}=+\infty$. If the conclusion of Lemma 1 is false, we obtain that there exists a positive integer $n$ and a $t^{*} \in\left(t_{n-1}, t_{n}\right]$ such that $\min \left\{x\left(t^{*}\right), y\left(t^{*}\right)\right\}=0$ and $x(t)>0, y(t)>0$ for $t_{0}<t<t_{*}$. The first possibility is that $x\left(t^{*}\right)=0$ and $y\left(t^{*}\right)>0$. For this case, it follows from the first and third equation of model (5) that

$$
x\left(t^{*}\right) \geq(1-p)^{n-1} x\left(t_{0}^{+}\right) \exp \left\{-\int_{t_{0}}^{t^{*}}[b+(\lambda-\varepsilon) y(\tau)] \mathrm{d} \tau\right\} \geq 0 .
$$

which contradicts the fact that $x\left(t^{*}\right)=0$.

The other case is that $x\left(t^{*}\right)>0$ and $y\left(t^{*}\right)=0$. In this regard, it follows from the second and fourth equation of model (5) that

$$
y\left(t^{*}\right) \geq y\left(t_{0}^{+}\right) \exp \left\{\int_{t_{0}}^{t^{*}}[\lambda x(\tau)-(b+c+\varepsilon)] \mathrm{d} \tau\right\} \geq 0 .
$$

which lead to a contradiction with $y\left(t^{*}\right)=0$. Therefore, according to above discussion, we can obtain that $x(t) \geq 0$ and $y(t) \geq 0$ for all $t \geq t_{0}$. This proof is complete.

In order to address the dynamical behaviors of model (5), we could construct two sections to the vector field of model (5) by

$$
\Sigma_{p}:=\{(x(t), y(t)): x(t)=(1-p) H, 0<y(t)<1-(1-p) H\},
$$

and

$$
\Sigma_{H}:=\{(x(t), y(t)): x(t)=H, 0<y(t)<1-H\} .
$$

Choosing section $\Sigma_{p}$ as a Poincaré section. Assume that for any point $P_{1}\left(H, y_{1}\right) \in \Sigma_{H}$, the trajectory $O^{+}\left(P_{1}, t_{0}\right)$ starting from the initial point $P_{1}\left(H, y_{1}\right)$ intersects section $\Sigma_{H}$ infinitely many times. That is, trajectory $O^{+}\left(P_{1}, t_{0}\right)$ jumps to section $\Sigma_{p}$ at point $P_{1}^{+}\left((1-p) H, y_{1}^{+}\right)$due to pulse effect. Moreover, trajectory $O^{+}\left(P_{1}, t_{0}\right)$ will reach at section $\Sigma_{H}$ at point $P_{2}\left(H, y_{2}\right)$, and then jumps to point $P_{2}^{+}\left((1-p) H, y_{2}^{+}\right)$on section $\Sigma_{p}$. Repeating this procedure, we get two pulse point sequences $\left\{P_{n}^{+}\left((1-p) H, y_{n}^{+}\right)\right\}$and $\left\{P_{n}\left(H, y_{n}\right)\right\} \quad(n=1,2, \cdots)$, where $y_{n+1}^{+}$is only determined by $y_{n}^{+}, H$, and $p$. Therefore, we can define a Poincaré map of section $\Sigma_{p}$ as

$$
y_{n+1}^{+}:=\mathcal{F}\left(y_{n}^{+}, H, p\right) .
$$

From the definition of Poincaré map $\mathcal{F}$, it easy to get that

$$
y_{n+1}^{+}=\mathcal{F}\left(y_{n}^{+}, H, p\right)=\mathcal{F}\left(\mathcal{F}\left(y_{n-1}^{+}, H, p\right)\right)=\mathcal{F}^{2}\left(y_{n-1}^{+}, H, p\right)=\cdots=\mathcal{F}^{n}\left(y_{1}^{+}, H, p\right) .
$$

Obviously, function $\mathcal{F}$ is continuously differential according to the CauchyLipschitz theorem. If there exist positive integer $k$ such that $y_{k+1}^{+}=\mathcal{F}^{k}\left(y_{1}, H, p\right)$, then trajectory $\mathrm{O}^{+}\left(P_{1}^{+}, t_{0}\right)$ of model (5) is said to be order- $k$ periodic solution. 


\section{Main Results}

Our main purpose in this section is to investigate the existence and orbital stability of periodic solution of model (5). From the geometrical construction of phase space of model (5), we note that the trajectory $O^{+}\left(M, t_{0}\right)$ from any initial point $M \in \Sigma_{p}$ intersects section $\Sigma_{H}$ infinite times with $H \leq x_{e}$. However, if $(1-p) H<x_{e}<H<1$, then trajectory $O^{+}\left(M, t_{0}\right)$ from any initial point $M \in \Sigma_{p}$ may be free from pulse effects or intersects section $\Sigma_{p}$ infinitely times, which depend on the initial conditions. Consequently, based on different positions of section $\Sigma_{H}$ we need to discuss the existence and orbital stability of periodic solution of model (5) in the cases of $H \leq x_{e}$ and $(1-p) H<x_{e}<H<1$.

Case I: The case of $H \leq x_{e}$.

For this case, it will prove that model (5) possesses a disease-free periodic solution, which is orbitally asymptotically stable.

Suppose $y(t)=0$ for all $t \in(0,+\infty)$, then model (5) degenerates into the following model

$$
\begin{cases}\frac{\mathrm{d} x(t)}{\mathrm{d} t}=b-b x(t)+e(1-x(t))+\delta x(t)(1-x(t)), & x(t)<H, \\ x\left(t^{+}\right)=(1-p) x(t), & x(t)=H .\end{cases}
$$

Integrating the first equation of model (7) with the initial condition $x(0)=x_{0}=(1-p) H$, one yields

$$
\tilde{x}(t)=\frac{\exp [(b+e+\delta)(t+C)]-b-e}{\delta+\exp [(t+C)(b+e+\delta)]},
$$

where

$$
C=\frac{1}{b+e+\delta} \ln \frac{(1-p) H \delta+b+e}{1-(1-p) H} .
$$

Assume that $x(T)=H$ and $x\left(T^{+}\right)=x_{0}=(1-p) H$, then we obtain

$$
T=\frac{1}{b+e+\delta} \ln \frac{(\delta H+b+e)(1-(1-p) H)}{(1-H)((1-p) H \delta+b+e)} .
$$

Therefore, model (5) possesses the following disease-free periodic solution, denoted by

$$
\left\{\begin{array}{l}
\phi_{1}(t)=\frac{\exp [(b+e+\delta)((t-(k-1) T)+C)]-b-e}{\delta+\exp [((t-(k-1) T)+C)(b+e+\delta)]}, \\
\phi_{2}(t)=0 .
\end{array}\right.
$$

where $t \in((k-1) T, k T], k=1,2, \cdots$.

On the stability of this disease-free periodic solution $\left(\phi_{1}(t), \phi_{2}(t)\right)$ we have the following result.

Theorem 2. For any $p \in(0,1)$ and $H \leq x_{e}$ the disease-free periodic solution (8) 
of model (5) is orbitally asymptotically stable.

Proof. We assume that section $\Sigma_{p}$ intersects line $L_{2}: x+y=1$ and $x$ axis at points $P$ and $Q$, respectively. From the geometrical structure of phase space of model (5), we know that trajectory starts from any point on set $\overline{P Q}=\{(x, y): x=(1-p) H, 0 \leq y \leq 1-(1-p) H\}$ will enter set $\overline{P Q}$. Further, set $\overline{P Q}=\{(x, y): x=(1-p) H, 0 \leq y \leq 1-(1-p) H\}$ is mapped to set $\overline{P_{1} Q}=\left\{(x, y): x=(1-p) H, 0<y<y_{P_{1}}\right\}$ by Poincaré map (6), where $y_{P}>y_{P_{1}}>0$. Then, set $\overline{P_{1} Q}$ is mapped to set $\overline{P_{2} Q}=\left\{(x, y): x=(1-p) H, 0<y<y_{P_{2}}\right\}$ and $y_{P}>y_{P_{1}}>y_{P_{2}}>0$. Repeat above-mentioned procedure, we gain one point sequences $\left\{y_{P_{i}}\right\}$ and which satisfy

$$
y_{P}>y_{P_{1}}>y_{P_{2}}>\cdots>y_{P_{n}}>\cdots>0,
$$

and

$$
\overline{\mid P Q}\left|>\overline{\mid P_{1} Q}\right|>\overline{\left|P_{2} Q\right|}>\cdots>\left|\overline{\mid P_{n} Q}\right|>\cdots>0,
$$

where $y_{P}=1-(1-p) H$.

From (9), it is concluded that the point sequence $\overline{\mid P_{n} Q}$ is monotonically decrease in the interval $\left(0, y_{P}\right]$ and converge to a fixed point in this bound region. That is $\lim _{n \rightarrow \infty} \overline{\left|P_{n} Q\right|}=0$.

Suppose that $(\bar{x}(t), \bar{y}(t))$ is a solution of small-amplitude perturbation of diseasefree periodic solution $\left(\phi_{1}(t), \phi_{2}(t)\right)$ with initial value $(\bar{x}(0), \bar{y}(0))=\left((1-p) H, \bar{y}_{0}\right)$, which first intersects section $\Sigma_{H}$ at point $\left(H, \bar{y}_{1}\right)$ and then jumps to point $\left((1-p) H, \bar{y}_{1}^{+}\right)$. Further, solution $(\bar{x}(t), \bar{y}(t))$ insects section $\Sigma_{H}$ at point $\left(H, \bar{y}_{2}\right)$ again. Repeating the above process, we have two point sequences $\left\{\bar{y}_{n}^{+}\right\}$and $\left\{\bar{y}_{n}\right\}$, where $\bar{y}_{n}^{+}=\bar{y}_{n}$. Furthermore, by $\lim _{n \rightarrow \infty} \mid \overline{P_{n} Q}=0$, it is clear that $\lim _{t \rightarrow \infty} \bar{y}(t)=0$. This shows that the disease-free periodic solution (8) of model (5) is orbitally asymptotically stable. This proof is complete.

Case II: The case of $(1-p) H<x_{e}<H<1$.

For this case, we know that there a point $E_{0}\left((1-p) H, y_{E_{0}}\right) \in \Sigma_{p}$ such that trajectory $O^{+}\left(E_{0}, t_{0}\right)$ is tangent to section $\Sigma_{H}$ at the point $E_{1}(H,(1-H)(b+e+\delta H)) /((\lambda-\varepsilon+\delta) H+e)$. Then the point $E_{1}$ is jump to the point $E_{1}^{+}((1-p) H,(1-H)(b+e+\delta H)) /((\lambda-\varepsilon+\delta) H+e)$ on section $\Sigma_{p}$ after pulse effect. According to the different positions of point $E_{1}^{+}$we has the following results.

Theorem 3. For any $p \in(0,1)$ and $(1-p) H<x_{e}<H<1$, if $y_{E_{0}}=(1-H)(b+e+\delta H) /((\lambda-\varepsilon+\delta) H+e)$, then model (5) exists a positive order-1 periodic solution. Further, if

$$
y_{E_{0}}>\frac{(1-H)(b+e+\delta H)}{(\lambda-\varepsilon+\delta) H+e},
$$

then model (5) exists a disease-free periodic solution (8), which is orbitally asymptotically stable.

For this case, (8) is a disease-free periodic solution of model (5), and the proof of stability is similar to the proof of Theorem 2, we therefore omit here. 


\section{Concluding Remarks}

In order to explore the effects of the state-dependent pulse control strategies on the transmission of the infectious diseases in a population of varying size, an SIRS epidemic model with varying total population and state-dependent pulse control strategy is proposed and analyzed in this paper. Theoretically analyzing this control model, we find that a disease-free periodic solution always exists and orbitally stable when condition $H \leq x_{e}$ holds. Theoretical results shows that the disease finally disappears if we control the fraction of susceptible individuals in relatively low levels. Furthermore, we obtained some sufficient condition on existence and stability of the positive order-1 periodic solution when $(1-p) H<x_{e}<H<1$. This amounts to that we can control the fraction of susceptible individuals and infected individuals within a retain range for a long time by appropriately choose the immune strength $p$ and critical threshold $H$. Therefore, we can concluded that state-dependent pulse vaccination is a feasible, economic, and high efficient method to prevention and control spread of diseases.

\section{Conflict of Interests}

The authors declare that there is no conflict of interests regarding the publication of this paper.

\section{Fund}

This research has been partially supported by the Natural Science Foundation of Xinjiang (Grant no. 2016D01C046).

\section{References}

[1] Hu, Z., Ma, W. and Ruan, S. (2012) Analysis of SIR Epidemic Models with Nonlinear Incidence Rate and Treatment. Mathematical Biosciences, 238, 12-20. http://dx.doi.org/10.1016/j.mbs.2012.03.010

[2] Meng, X. and Chen, L. (2008) The Dynamics of a New SIR Epidemic Model Concerning Pulse Vaccination Strategy. Applied Mathematics and Computation, 197, 582-597. http://dx.doi.org/10.1016/j.amc.2007.07.083

[3] Zhang, T., Meng, X., Liu, R. and Zhang, T. (2014) Periodic Solution of a Pest Management Gompertz Model with Impulsive State Feedback Control. Nonlinear Dynamics, 78, 921938. http://dx.doi.org/10.1007/s11071-014-1486-y

[4] Tang, B., Xiao, Y., Tang, S. and Cheke, R. (2016) A Feedback Control Model of Comprehensive Therapy for Treating Immunogenic Tumours. International Journal of Bifurcation and Chaos, 26, $22 \mathrm{p}$.

[5] Tang, S., Tang, B., Wang, A. and Xiao, Y. (2015) Holling II Predator-Prey Impulsive Semi-Dynamic Model with Complex Poincaré Map. Nonlinear Dynamics, 81, 1575-1596. http://dx.doi.org/10.1007/s11071-015-2092-3

[6] Nie, L., Teng, Z. and Toores, A. (2012) Dynamics Analysis of an SIR Epidemic Model with State Dependent Pulse Vaccination. Nonlinear Analysis: Real World Applications, 13, 1621-1629. http://dx.doi.org/10.1016/j.nonrwa.2011.11.019

[7] Tang, S., Xiao, Y. and Clancy, D. (2005) New Modelling Approach Concerning Integrated Disease Control and Cost-Effectivity. Nonlinear Analysis: Theory, Methods and Applica- 
tions, 63, 439-471. http://dx.doi.org/10.1016/j.na.2005.05.029

[8] Yang, W., Sun, C. and Arino, J. (2010) Global Analysis for a General Epidemiological Model with Vaccination and Varying Population. Journal of Mathematical Analysis and Applications, 372, 208-223. http://dx.doi.org/10.1016/j.jmaa.2010.07.017

[9] Hui, J. and Zhu, D. (2007) Dynamics of SEIS Epidemic Models with Varying Population Size. International Journal of Bifurcation and Chaos, 15, 1513-1529. http://dx.doi.org/10.1142/S0218127407017902

[10] Li, J. and Ma, Z. (2002) Qualitative Analyses of SIS Epidemic Model with Vaccination and Varying Total Population Size. Mathematical and Computer Modelling, 35, 1235-1243. http://dx.doi.org/10.1016/S0895-7177(02)00082-1

[11] Naresh, R. and Tripathi, A. (2005) Modelling and Analysis of HIV-TB Co-Infection in a Variable Size Population. Mathematical Modelling and Analysis, 10, 275-286.

[12] Busenberg, S. and van den Driessche, P. (1990) Analysis of a Disease Transmission Model in a Population with Varying Size. Journal of Mathematical Biology, 28, 257-270. http://dx.doi.org/10.1007/BF00178776

[13] Lakshmikantham, V., Bainov, D. and Simeonov, P. (1989) Theory of Impulsive Differential Equations. World Scientific, Singapore. http://dx.doi.org/10.1142/0906

Submit or recommend next manuscript to SCIRP and we will provide best service for you:

Accepting pre-submission inquiries through Email, Facebook, LinkedIn, Twitter, etc.

A wide selection of journals (inclusive of 9 subjects, more than 200 journals)

Providing 24-hour high-quality service

User-friendly online submission system

Fair and swift peer-review system

Efficient typesetting and proofreading procedure

Display of the result of downloads and visits, as well as the number of cited articles

Maximum dissemination of your research work

Submit your manuscript at: http://papersubmission.scirp.org/

Or contact jamp@scirp.org 